\title{
Spatially Varied Control Channel Assignment in Cognitive Radio Ad Hoc Networks
}

\author{
Hsiu Yu \\ Institute of Computer and Communication Engineering \\ National Cheng Kung University \\ Tainan, Taiwan 70101 \\ andrea@dcl.ee.ncku.edu.tw
}

\author{
Kuo-Feng Ssu \\ Department of Electrical Engineering \\ National Cheng Kung University \\ Tainan, Taiwan 70101 \\ ssu@ee.ncku.edu.tw
}

\begin{abstract}
In order to ease the control message exchange, a constantly available control channel is demanded in cognitive radio ad hoc networks. Due to the characteristics of the cognitive radio network, available channel sets vary with both time and space. A globally fixed in-band control channel is not always ready to use, so a local and dynamic control channel is more preferred. In this paper, a spatially varied control channel assignment scheme, called SVC, is developed. SVC takes advantage of the spatial similarity among the available channel sets which neighboring secondary users hold. The secondary users believe that their neighbors would have the same control channel choices as theirs if they value the channels in the same way. Each user can observe the environment and make the decision independently. As a result, SVC is scalable and has the ability to recover immediately from the sudden appearance of primary users. Besides, a simple neighbor discovery mechanism is also introduced to solve the problem that most of the control channel assignment schemes require neighbor information in advance.
\end{abstract}

\section{INTRODUCTION}

The cognitive radio network is a promising solution to the spectrum under-utilization and scarcity problem [1]. There are two types of users in the network, including primary users and secondary users. The primary users hold the spectrum licenses. The secondary users can access the spectrum under the premise that the primary users are not affected by their operations. Thus, the secondary users should have the abilities to detect the activities of primary users and choose the available spectrum dynamically to meet their transmission requirements.

To exchange control messages and coordinate spectrum without difficulties, an always available control channel is needed. In the conventional multi-channel systems, the control channels are in-band and globally common. However, in the cognitive radio network, since the secondary users can only use channels without influences on the primary users, the available channel sets differ from each other. The existence of globally in-band common channels in the cognitive radio network cannot be guaranteed [2]. Some approaches assign out-of-band control channels alternatively, but using out-ofband dedicated control channel not only has the spectrum scarcity problem but security issues. If the cognitive radio network is constructed on the battle field, attackers could perform the jamming attack on the control channel and then crash the network [2]. Some approaches do not use control channels [3], [4]. They just hop among channels to meet neighbors. Each secondary user is assigned a channel every time slot [3]. If the secondary user has nothing to transmit, the user stays on the assigned channel (home channel) to listen. If the user has packets to transmit, the user will compute the possible home channel of the receiver, switch to that channel, and transmit packets directly. Obviously, the approaches without control channels have larger delay and less successful transmissions.

Due to the correlation between the available channel sets of secondary users and the activities of primary users, the available channel sets have spatial and temporal similarities. Even though it is hard to find a global common channel, a large amount of local common channels exist among neighboring secondary users. The control channel can be selected from these local common channels [2], [5], [6], [7], [8]. Secondary users make their best efforts to choose the same control channel as those of their neighbors. Nonetheless, neighboring secondary users might use different control channels. The secondary users on the same control channel are called members in the same cluster. They can find each other directly on the control channel. The secondary users should switch to the control channels of neighbors or the channels which they and their neighbors own jointly whenever they want to contact their neighbors in different clusters. Therefore, to reduce the overhead of transmission, the main goal of local control channel assignment approaches is forming clusters as large as possible. Then, the number of secondary users around cluster boundaries can be reduced. In addition to the cluster size, efficient recovery is also an important issue. In the cognitive radio network, secondary users should stop using the control channels right away if the primary users are active. To maintain the transmission qualities of secondary users, new control channels should be determined immediately. Besides, selecting the local common control channel needs the neighbor information but exchanging the neighbor information efficiently needs the control channel. Thus, a control channel assignment scheme with the neighbor discovery function is also necessary.

Based on the fact that the available channel sets are similar in time and space, a spatially varied control channel assignment scheme, called SVC, is proposed in this paper. The similar available channel sets have some identical characteristics, 
such as the smallest channel ID or the best channel quality in the set. If the secondary users select the control channel based on a given characteristic, the secondary users affected by the same primary user with that characteristic will make the same choice. Eventually, they choose control channels individually, but naturally reach a consensus. To elevate the constraint that the cluster sizes would be limited by the distribution of some primary users if the decisions are made only based on one characteristic, the second characteristic is introduced to expand clusters. Finally, a neighbor discovery mechanism is also provided based on the observation that secondary users can locate more neighbors on the channels with better qualities.

This paper has three main contributions. First, a simple and fast local control channel assignment scheme is developed. Second, because of the simplicity, the control channel can be determined immediately if the original control channel is occupied by the primary user. Finally, SVC does not rely on any other neighbor discovery mechanism. SVC solves the neighbor discovery problem at the same time.

\section{RELATED WORK}

There have been plenty of local control channel assignment researches in the recent years. CogMesh [8] is a clusterbased scheme. New secondary users initially scan all available channels and then decide to be cluster heads of new clusters or to join existing clusters. The cluster members must be the neighbors of the cluster head, so the cluster size is limited to the largest number of neighbors within the transmission range of the cluster head.

The idea of the swarm intelligence inspired by the collective behavior of the social insect, is also applied to the control channel assignment problem [6], [7]. SWARM [6] maps the secondary user to the agent, and the most recent control channel choices of neighbors to the pheromone. The secondary user first selects the best quality channel as the control channel and calculates the probabilities that each channel is selected as the control channel. The secondary user then broadcasts his choice and the according quality. Whenever the user knows the choices of neighbors, the probabilities for each channel will be updated. If the channel is the control channel of the neighbor, it obtains a positive reward of the probability to be choosen. Otherwise, it receives a negative reward. In the end of each cycle, the secondary user chooses the channel with the largest probability as the control channel. When the available channels have fair qualities, the cluster sizes can not be large. The control channel choices of secondary users can not be stable, neither.

Some approaches assign sets of control channels to secondary users to maintain the connectivities [2], [5]. The new secondary user initially collects the information about the available channel sets of neighbors, and then iteratively selects a channel, which results in the best connectivity for the secondary user and its remaining neighbors, as the control channel until every neighbor has a common control channel with it [2]. The control overhead is heavy and the relatively long time is taken to find a control channel set.

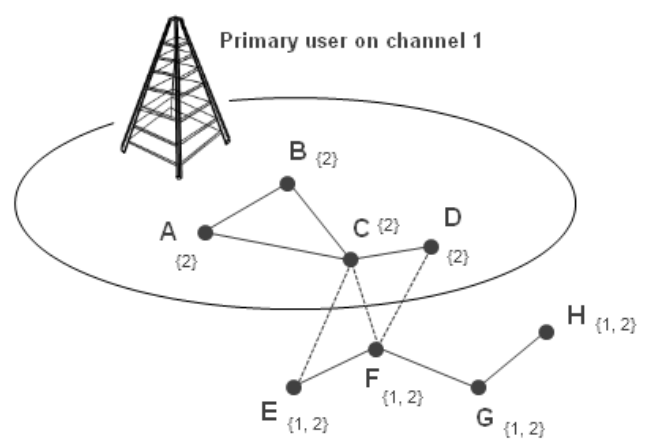

Fig. 1: The user chooses the smallest ID channel in the available channel set as the control channel. A, B, C, and D use channel 2 as the control channel. The control channel of the other users is channel 1 .

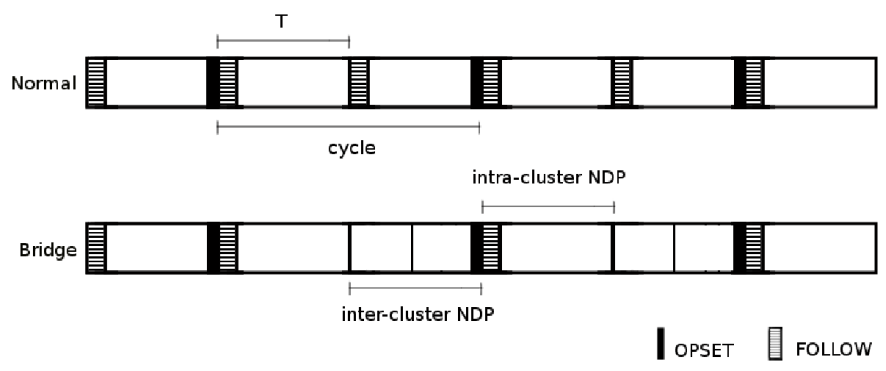

Fig. 2: Working cycles.

\section{SySTEM FRAMEWORK}

The network is constructed by two types of users, primary users and secondary users. They are called PUs and users in this paper for short. Users can access a given spectrum only when the PUs of the spectrum do not use it. It is assumed that the users are aware of the available channels and their associated channel qualities with some spectrum sensing mechanisms [9], [10]. The available channel sets of users are different but of similarities in time and space due to the activities of PUs. The users in the network communicate with each other through ad hoc connections. They coordinate data channels and exchange routing information on their local control channels.

Control channels are chosen based on the local observations of users. The available channel sets of users are spatially similar. If a user chooses a channel as the control channel according to some characteristics of the available channel set, it is very likely to use the same control channel as those of its neighbors. As a result, some users may form a cluster naturally because of the choices of control channels. In the following paper, users that have the same control channel and can directly transmit control messages without channel switch are called members in the same cluster. The control channels which users choose independently are not global, so there are two types of transmission, intra-cluster transmission and intercluster transmission. Inter-cluster transmission occurs on the boundaries of the clusters. Inter-cluster transmission spend 
more time and energy than intra-cluster transmission. For improving transmission delay, the goal of SVC is to minimize the number of clusters and form the clusters as large as possible.

Users could operate in either the normal mode or the bridge mode. When a user detects that all his neighbors are intra-cluster neighbors, the user works in the normal mode. Otherwise, the user operates in the bridge mode. As illustrated in Fig. 1, A, B, G, and $\mathrm{H}$ are normal users, whereas C, D, E, and $\mathrm{F}$ are bridge users. Bridge users periodically invite intercluster neighbors to the cluster where they belong. Time is divided into cycles. As shown in Fig. 2, a cycle is further separated into two periods with length $T$. The former period is the intra-cluster neighbor discovery period, while the later period is the inter-cluster neighbor discovery period. Users in different modes have different working cycle. They broadcast FOLLOW messages to inform neighbors that they are alive and also invite inter-cluster neighbors to their clusters. The FOLLOW massage includes the user identifier, the channel identifier, the operation mode, the father identifier, and the cycle start time. The father identifier field is only used in the initialization phase. The normal user only broadcasts a FOLLOW message every period on his control channel. The bridge user broadcasts a FOLLOW message at the beginning of the intra-cluster NDP on his control channel and broadcasts FOLLOW messages at the head and the middle of the intercluster NDP on his two best channels. At the end of the cycle, users in both modes reset the operation mode in terms of the neighbor information they collect in the cycle. Moreover, the bridge user might change the control channel to join the neighbor cluster.

\section{SPATIAlly VARied Control Channel Assignment}

Users discover neighbors, select an operation mode, and migrate from one control channel to the other according to the predefined working cycle of each mode. Users should set their operation modes first, and then could discover neighbors based on their schedules. However, the operation mode is determined with the neighbor information. Therefore, a rough mode selection mechanism should also be provided for the users who are new in the network. Initialization, neighbor discovery, mode selection, and control channel migration will be described in details.

\section{A. Initialization}

Due to similarities of available channel sets, the control channel which the user chooses based on a specific channel characteristic will have a great possibility to be the same as those of its neighbors. For simplicity, the user sets the lowest ID channel in the available channel set as its control channel in the initialization phase. Obviously, those users who have control channels with poorer qualities are more likely to be on the boundaries of the clusters, so they are more suitable to be in the bridge mode initially.

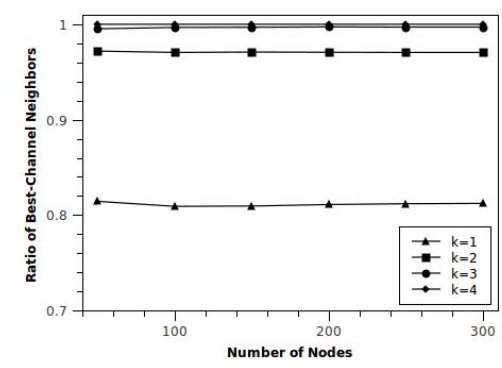

Fig. 3: Probability to find neighbors on best $\mathrm{k}$ channels.

After choosing the control channel, the user listens on the control channel for $T$. If the user hears nothing during this period, the user waits additional $I / Q$, broadcasts a FOLLOW message, then starts a new working cycle as a bridge user. The father identifier is the same as its user identifier and the cycle start time is the current time in the FOLLOW message. $I$ is a relatively small period. $Q$ is the quality of the smallest id channel. The user have more chances to be the founder of the mode-initialization action, if the quality of his control channel is better then those of his neighbors. On the other hand, if the user receives a FOLLOW message during the listening period, the user will broadcast a FOLLOW message immediately and wait for a while. The father identifier of the FOLLOW message is set to be the sender of the FOLLOW message just received. After that, if the user receives a FOLLOW message and the father identifier is himself, the user will enter the normal mode. Otherwise, the user will operate in the bridge mode in the next cycle. The user is probably on the boundaries of the cluster since the user does not receive any reply.

\section{B. Neighbor Discovery}

The operation cycle is separated into two periods. The first period is the intra-cluster NDP and the second period is the inter-cluster NDP. The user broadcasts a FOLLOW message on the control channel when the user enters the intra-cluster NDP. The user replies a FOLLOW message immediately if the user receives a FOLLOW message. It is noted that users in the network are not synchronized. Nevertheless, users can receive all the FOLLOW messages from their neighbors. Because all users wait $T / 2$ plus $I / Q$ at most in the initialization phase, the difference of times that users broadcast FOLLOW messages at the beginning of a cycle on the control channel does not exceed $T / 2$. The normal user only broadcasts a FOLLOW message again at the beginning of the inter-cluster neighbor discovery period on the control channel. The intercluster neighbor discovery period for bridge users is further divided into two parts. The bridge user broadcasts a FOLLOW message on the channel with the best quality in the former part and broadcasts a FOLLOW message on the second-quality channel during the later part. Since the difference between the start times of users is less than $T / 2$, the former part of the inter-cluster NDP of one user must overlap the later part of the other user. In addition, from the simulation result shown in 
Fig. 3, a user can reach almost all of his neighbors on best $k$ channels. Using the previous described inter-cluster neighbor discovery mechanism, the user can find inter-cluster neighbors on the two best channels. After one cycle, the user can know almost all neighbors. The user sets the mode and the control channel for the next cycle based on the neighbor information collected in this cycle.

\section{Mode Selection}

In the end of the cycle, users reset the operation mode for the next cycle. The normal user changes into the bridge mode if the user receives a FOLLOW message from one of his intercluster neighbors. Such situation happens when the user is new to the network or when some of his intra-cluster neighbors just migrate to the other clusters. If the user just arrives in the network, the user may choose the improper mode in the initialization phase. If the user has neighbors in the bridge mode, those neighbors might migrate to the neighbor cluster due to the better control channel quality the neighbor cluster has. On the other hand, the bridge user might change into the normal mode, if all his neighbors become intra-cluster neighbors. In other words, the user and his neighbors set the same channel as the control channel. This happens when the user has a control channel with the better quality.

\section{Control Channel Migration}

Since the operation in the bridge mode spends more energy, users incline to be in the normal mode. In addition, users can reach more neighbors on the channel with the better quality so that they have larger probabilities to be in the normal mode. When the neighbor cluster has the better-quality available channel as the control channel, the user would migrate to the neighbor cluster and invite other neighbors to join that cluster. If his neighbors can be the intra-cluster neighbors, the user can be in the normal mode in the next cycle. It is noted that control channel migration only occurs to bridge users because they would receive the FOLLOW message from inter-cluster neighbors and be aware of neighbor clusters nearby.

Furthermore, to break the loops among users with similarquality control channels and to avoid clusters extending on one side and contracting on the other side, users only migrate to larger ID channels. For the better opportunity to be a normal user, once the user finds that one of his neighbors has a control channel with the larger ID, the better quality, and the availability, the user would migrate to the control channel which the neighbor uses currently. After a few cycles, the network will become stable. Some clusters are expanded and the number of bridge users is decreased.

\section{E. Transmission}

Bridge users play active roles in the network. They are responsible for not only extending the clusters but delivering messages across clusters. Transmission in the network can be categorized into intra-cluster transmission and inter-cluster transmission. Transmission inside a cluster occurs to normal users. They coordinate data channels on the control channel
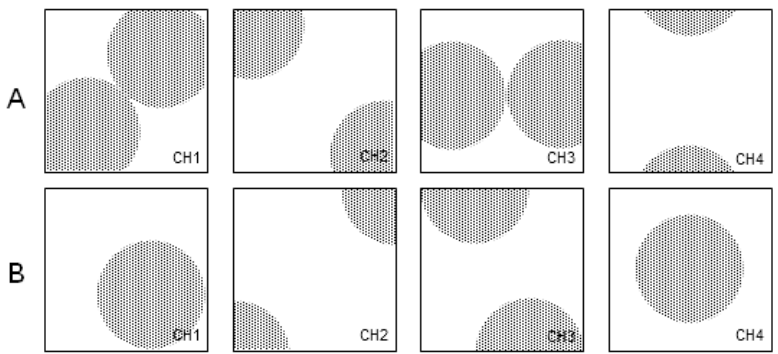

Fig. 4: Two scenarios are considered in the simulation. The total number of channels in one scenario is four. There are two PUs on each channel. The shaded areas are the transmission ranges of the PUs. The PUs that are not shown in the figures do not affect the users in the interested area.

whenever they have packets to send. Transmission between clusters is more complex. In Fig. 1, messages sending from A to $\mathrm{H}$ must go through the bridge user $\mathrm{C}$, the bridge user $\mathrm{F}$, and then the normal user $\mathrm{G}$. The same as the intra-cluster case, users should coordinate data channels before sending data. However, they cannot accomplish this anytime. When a normal user needs to transmit a packet to a bridge user which is currently not on the control channel, the only thing it can do is waiting for the bridge user to switch back. While a bridge user has a packet to send, it can switch to the channel where the receiver stays if the channel is accessible to it at that moment. Otherwise, it should wait until the receiver switches to an accessible channel.

\section{Simulation Results}

The simulation is setup as follows. Users are randomly deployed in a $600 \mathrm{~m} \times 600 \mathrm{~m}$ area. They share four licensed channels with 8 PUs. The transmission range of a PU is $200 \mathrm{~m}$ and that of a user is $100 \mathrm{~m}$. Two scenarios are considered as shown in Fig. 4. In the scenario A, the available channels for each user are of similar qualities. In the scenario B, the diversity of the channel qualities is wider. Two mechanisms described previously, HD-MAC [2] and SWARM [6], are selected for performance comparison.

The comparison of the average sizes of clusters where users belong under various total numbers of users is shown in Fig. 5. The average cluster size is normalized with the total number of users in the network. When the number of users in a fixed size area grows, more users are affected by a PU and thus the size of the cluster increases. Therefore, the growth of the network density has no influence on the normalized average cluster sizes of all mechanisms except for the 50-user case. When the network is too sparse, users experience weak connectivity. Since the neighbors of users are few, the clusters cannot be enlarged properly. In the scenario A, SVC produces larger clusters. The average size of clusters is about half the users in the network. At the same time, SWARM performs the worst. The average cluster size is less than one-third of users. It comes from the fact that the probabilities to choose a channel as the control channel are similar, since the qualities of 


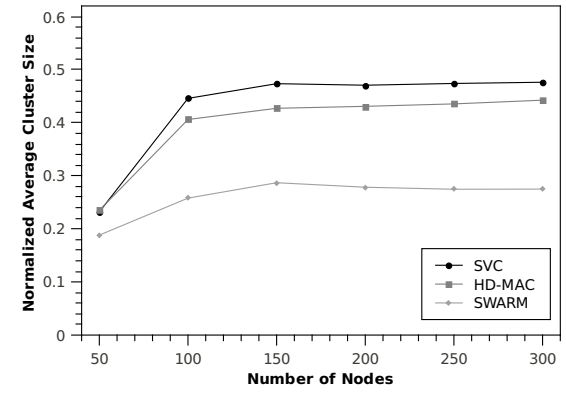

(a) Scenario $\mathrm{A}$.

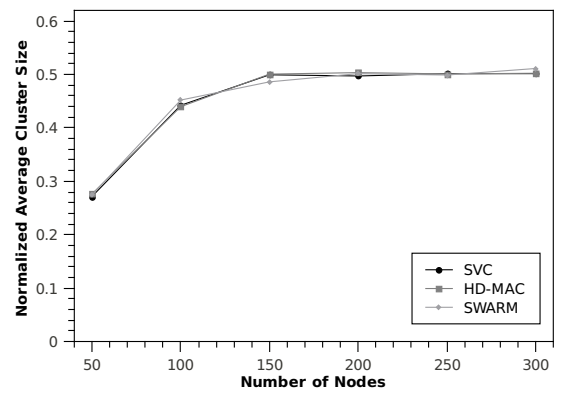

(b) Scenario B.

Fig. 5: Normalized average cluster size.

available channels are similar. Therefore, SWARM produces clusters with similarly small sizes while SVC expands some clusters successfully. In the scenario B, all three schemes have similar performances.

Next, the distribution of 100 users to various-sized clusters is examined. Fig. 6a shows that when the qualities of available channels are about the same, the opportunities that users with SWARM locate in clusters with various sizes are almost the same. Half of the users choose the clusters with sizes less than 20. The size of the biggest cluster they can choose is about 50. SVC and HD-MAC perform equally. Particularly, HDMAC forms the biggest cluster whereas SVC suppresses the formation of small clusters. Fig. $6 \mathrm{~b}$ demonstrates performance with the larger diversity of the available channel qualities. SWARM forms the biggest cluster, but produces lots of fragmentary clusters. On the other hand, both SVC and HDMAC are able to form the large clusters and also reduce the number of small clusters.

\section{CONCLUSION}

To facilitate the network operation, such as routing and maintaining the network topology, the control channel is necessary in the cognitive radio ad hoc network. This paper presented a spatially varied control channel assignment (SVC) scheme. Taking advantage of the similarity of available channel sets, users can choose control channels individually. Thus, the users can reconstruct new control channels immediately if the activities of primary users happen. The SVC not only assigns control channels efficiently but also enables neighbor discovery. Simulation results have shown that the SVC scheme

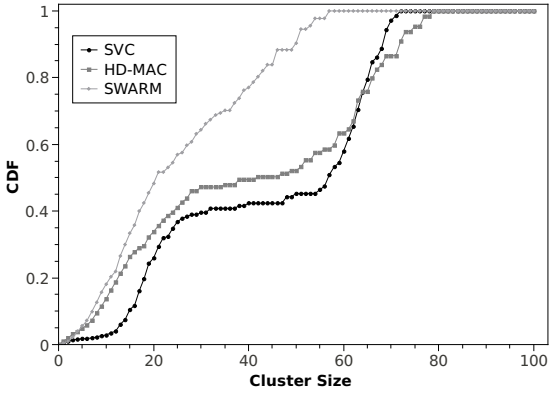

(a) Scenario A.

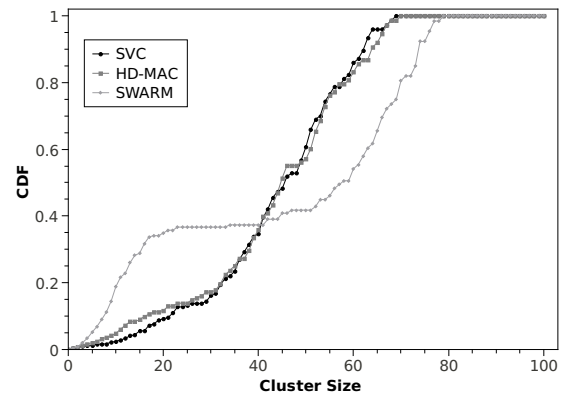

(b) Scenario B.

Fig. 6: Cumulative distribution function of cluster sizes.

is suitable for both scenarios and performs as good as the more complicated scheme, such as HD-MAC.

\section{REFERENCES}

[1] I. F. Akyildiz, W. Y. Lee, M. C. Vuran, and S. Mohanty, "Next generation/dynamic spectrum access/cognitive radio wireless networks: a survey," Comput. Netw., vol. 50, no. 13, pp. 2127-2159, Sept. 2006.

[2] J. Zhao, H. Zheng, and G. H. Yang, "Spectrum sharing through distributed coordination in dynamic spectrum access networks," Wirel. Commun. Mob. Comput., vol. 7, no. 9, pp. 1061-1075, Nov. 2007.

[3] C. Xin and X. Cao, "A cognitive radio network architecture withou control channel," in IEEE Global Telecommunications Conference, Dec. 2009, pp. 1-6.

[4] C. Cormio and K. R. Chowdhury, "Common control channel design for cognitive radio wireless ad hoc networks using adaptive frequency hopping," Ad Hoc Netw., vol. 8, no. 4, pp. 430-438, June 2010.

[5] B. F. Lo, I. F. Akyildiz, and A. M. Al-Dhelaan, "Efficient recovery control channel design in cognitive radio ad hoc networks," IEEE Trans. Veh. Technol., vol. 59, no. 9, pp. 4513-4526, Nov. 2010.

[6] T. Chen, H. Zhang, M. D. Katz, and Z. Zhou, "Swarm intelligence based dynamic control channel assignment in CogMesh," in IEEE International Conference on Communications Workshops, May 2008, pp. $123-128$.

[7] C. Doerr, D. C. Sicker, and D. Grunwald, "Dynamic control channel assignment in cognitive radio networks using swarm intelligence," in IEEE Global Communications Conference, Dec. 2008, pp. 1-6.

[8] T. Chen, H. Zhang, G. M. Maggio, and I. Chlamtac, "CogMesh: A cluster-based cognitive radio network," in IEEE International Symposium on New Frontiers in Dynamic Spectrum Access Networks, Apr. 2007, pp. $168-178$.

[9] W. Saad, Z. Han, M. Debbah, A. Hjorungnes, and T. Basar, "Coalitional games for distributed collaborative spectrum sensing in cognitive radio networks," in IEEE International Conference on Computer Communications, Apr. 2009, pp. 2114-2122.

[10] B. Shen, C. Zhao, and Z. Zhou, "User clusters based hierarchical cooperative spectrum sensing in cognitive radio networks," in International Conference on Cognitive Radio Oriented Wireless Networks and Communications, June 2009, pp. 1-6. 